\title{
Substituição da Insulina NPH por Insulina Glargina em Uma Coorte de Pacientes Diabéticos: Estudo Observacional
}

\section{artigo original}

\author{
FREDERICO F.R. MAIA \\ FABRÍCIO J. MELO \\ ISABELLA M. ARAÚJo \\ LeVimar R. ARaújo
}

\author{
Departamento de Fisiologia, \\ Faculdade de Ciências Médicas \\ de Minas Gerais - FCMMG, e \\ Clínica de Endocrinologia e \\ Metabologia - Hospital \\ Universitário São José, FCMMG, \\ Belo Horizonte, MG.
}

Este estudo avaliou o impacto da introdução da insulina glargina na terapia basal/bólus em pacientes com diabetes mellitus do tipo 1 (DM1) e do tipo 2 (DM2), com controle inadequado ( $\mathrm{A} 1 \mathrm{c}>6,9 \%$ ) em uso prévio de insulina basal NPH. Foi realizado um estudo retrospectivo, não-controlado, com 49 pacientes $(28 \mathrm{~F} / 21 \mathrm{M})$, idade média $24,7 \pm 16,5$ anos, tempo de diabetes de $13,2 \pm 10,1$, sendo $93,1 \%$ dos pacientes com DM1, que receberam insulina glargina combinada com insulina ultra-rápida (aspart/lispro) pré-refeições, durante 90 dias de seguimento. Foram analisados dose total de insulina, incidência de hipoglicemias, crises convulsivas, complicações hiperglicêmicas (cetoacidose) e níveis de $A 1 \mathrm{c}$ antes e após três meses do uso da insulina glargina. Os valores de A1c foram determinados pelo método HPLC, com valores de referência de $4,3 \%$ a $6,9 \%$. Após 3 meses da modificação do esquema basal para insulina glargina, observou-se redução significativa dos níveis de $A 1 \mathrm{c}(10,2 \pm 2,0$ vs. $9,1 \pm 1,8 \% ; p=0,019$ ). Além disso, verificou-se redução de $0,11 \mathrm{U} / \mathrm{kg} / \mathrm{dia}$ na dose total de insulina utilizada ( $\mathrm{NPH}: 0,75 \mathrm{U} / \mathrm{kg}$ para $0,64 \mathrm{U} / \mathrm{kg}$ de insulina glargina; $\mathrm{p}<0,05$ ). $\mathrm{O}$ uso da insulina glargina associou-se com redução das crises hipoglicêmicas $(\mathrm{p}=$ $0,02)$, crises convulsivas por hipoglicemia grave $(p=0,023)$ e nenhum caso de cetoacidose $(p=0,001)$. Este estudo corrobora a eficácia da introdução da insulina glargina em pacientes diabéticos mal controlados, em uso prévio de insulina NPH, com redução importante dos níveis de A1c. Estima-se uma melhora da qualidade de vida desses pacientes, marcada pela redução de eventos hipoglicêmicos (inclusive os graves), episódios de cetoacidose e utilização de menor dose diária de insulina, com provável impacto em políticas de saúde pública. (Arq Bras Endocrinol Metab 2007;51/3:426-430)

Descritores: Insulina glargina / insulina NPH; Terapia basal / bólus; Diabetes mellitus; A1c.

\section{ABSTRACT}

Switching of NPH Insulin to Glargine Therapy in a Cohort of Poorly Controlled Diabetic Patients: Observational Study.

This study examined the impact of insulin glargine introduction in basal-bolus therapy in type 1 and type 2 diabetic patients with inadequate metabolic control (A1C > $6.9 \%)$ using previous NPH insulin regime. In this uncontrolled, retrospective study, 49 patients $(28 \mathrm{~F} / 21 \mathrm{M})$, average age $24.7 \pm 16.5$, mean duration of DM $13.2 \pm 10.1$ yrs., 93.1\% DM1 patients, received insulin glargine plus mealtime rapid-acting insulin (lispro or aspart) followed by 90 -day treatment. We analyzed mean total insulin dose, incidence of hypoglycemic events, convulsive crisis, hyperglycemic complications and A1c levels before and after three months of introduction of glargine therapy. A1c values were determined using the HPLC instrument, with a normal range of $4.3 \%$ to $6.9 \%$. After switching to insulin glargine therapy, mean A1c dropped from $10.2 \pm 2.0$ to 9.1 $\pm 1.8 \%$, with significant impact $(p=0.019)$. We observed a significant reduction of $0.11 \mathrm{U} / \mathrm{kg} / \mathrm{day}$ in total insulin dose, dropped from $0.75 \mathrm{U} / \mathrm{kg}$ of NPH to $0.64 \mathrm{U} / \mathrm{kg}$ of glargine, with significant correlation $(p<0.05)$. The introduction of glargine therapy was coincident with a decrease of hypoglycemic crisis $(p=0.02)$, convulsive events due to hypoglycemia (severe hypoglycemic crisis) $(p=0.023)$ and ketosis $(p=0.001)$ switching MDI-treated patients with improvement of metabolic control (reduction of A1c levels). This therapy improved quality of life in these patients due to a significant reduction of hypoglycemic (including severe) events, ketosis episodes and total daily insulin dose, with important impact on health public services. (Arq Bras Endocrinol Metab 2007;51/3:426-430)

Keywords: Glargine insulin / NPH insulin; Basal-bolus therapy; Diabetes mellitus; A1c
Recebido em 03/05/06

Revisado em 27/06/06 e 08/09/06 Aceito em 12/10/06 
$\mathrm{O}$ DIABETES MELLITUS (DM) É doença crônica, debilitante, que acomete cerca de 170 milhões de pessoas em todo o mundo (1), com repercussão sócio-econômica importante em função das complicações crônicas secundárias ao controle metabólico deficiente (2).

Atualmente, verifica-se no Brasil um baixo consumo de insulinas ultra-rápidas, sendo o esquema de insulina NPH, 2 vezes ao dia, o mais comumente utilizado (1). Apesar do aparecimento de novas drogas e melhor conhecimento da fisiopatologia do DM, o controle metabólico permanece insatisfatório na maioria da população diabética. Dados americanos mostram que menos da metade $(<50 \%)$ dos diabéticos mantém sua doença bem controlada (2).

A American Diabetes Association (ADA) recomenda uma glico-hemoglobina (Alc) $<6,5 \%$, glicemia pré-prandial $(\mathrm{FPG})<110 \mathrm{mg} / \mathrm{dL}$ e glicemia pós-prandial $(\mathrm{PPG})<140 \mathrm{mg} / \mathrm{dL}$, tolerável até $180 \mathrm{mg} / \mathrm{dl}$, como alvos de controle satisfatório $(3,4)$. Os grandes estudos, DCCT e UKPDS, mostraram que a redução de $1 \%$ na Alc influi significativamente na proteção à microangiopatia e à neuropatia. No entanto, estima-se que mesmo pacientes com níveis adequados de Alc experimentem episódios freqüentes de hipoglicemia noturna e hiperglicemia pós-prandial, possíveis complicadores da adesão ao tratamento intensivo e rígido (5).

Estudos recentes têm demonstrado a eficácia da insulina glargina, um análogo com ação de 24 h, no controle glicêmico de pacientes com DM tipo 1 (DM1) e tipo 2 (DM2) tratados previamente com insulina NPH (6-9). Estudos com clamp euglicêmico em pacientes saudáveis indicam que a absorção dessa insulina é prolongada $(24 \mathrm{~h})$ e sem picos $(10,11)$, com maior estabilidade glicêmica e menor incidência de hipoglicemias graves e hipoglicemia noturna, além de não ocasionar ganho de peso significativo.

Este estudo buscou avaliar o impacto da substituição da insulina NPH por insulina glargina em uma coorte de pacientes diabéticos mal controlados (Alc > $6,9)$, após três meses de tratamento. Buscou-se, ainda, avaliar o impacto dessa nova terapia sob os eventos hipoglicêmicos (inclusive graves), hiperglicêmicos (cetoacidose) e dose diária de insulina utilizada.

\section{CASUÍSTICA E MÉTODOS}

Foi realizado um estudo retrospectivo, não-controlado, em uma coorte de 49 pacientes diabéticos, atendidos pela Clínica de Endocrinologia e Metabologia do Hospital Universitário São José (CEM-HUSJ), no período de abril de 2003 a agosto de 2005, submetidos à mudança de terapêutica basal da insulina NPH para a insulina glargina.
Não houve restrição quanto a idade, sexo ou tempo de diabetes. Houve predomínio de pacientes com DMl $(93,1 \%)$ vs. DM2 $(6,9 \%)$. Os pacientes foram selecionados ao acaso, por cálculo amostral (grau de confiança de 90\%, erro máximo de $5 \%$ ) de um universo de 116 pacientes atendidos. O predomínio de DMl provavelmente se deu pela seleção ao acaso e por se tratar de ambulatório de referência.

Foram critérios de inclusão essenciais para participação no estudo: a presença de Alc > 6,9\%, sendo considerados metabolicamente descompensados, e estar em uso de insulina NPH humana combinada ou não com insulinas regular ou ultra-rápida (lispro/aspart) pré-refeição, por no mínimo 6 meses de tratamento.

Todos os pacientes foram submetidos previamente às orientações básicas de tratamento, orientações dietéticas, atividade física e, sobretudo, manuseio e cuidados com a insulina glargina. $\mathrm{O}$ ajuste das doses foi estabelecido pela equipe assistente, com base na titulação "LANMET" (12). A dose inicial foi de 10 UI de insulina glargina, seguida de ajuste a cada três dias (aumento de duas unidades, progressivamente) com alvo terapêutico de glicemia de jejum inferior a $100 \mathrm{mg} / \mathrm{dl}$. A glicemia capilar foi realizada um mínimo de 6 vezes ao dia, em horários pré-estabelecidos (pré e pós-prandiais, três vezes ao dia), além das suspeitas de hipo ou hiperglicemias. Os pacientes foram orientados a registrar os eventos glicêmicos ocorridos nesse período.

As variáveis analisadas foram: dose total de insulina (somatória da insulina basal e insulina em bólus com regular, lispro ou aspart, quando utilizadas), incidência de crises hipoglicêmicas, crises convulsivas por hipoglicemia grave, internações por cetoacidose (ou estado hiperosmolar nãocetótico) e níveis de Alc antes e após três meses da introdução da insulina glargina, sendo este o principal parâmetro de controle metabólico.

As excursões glicêmicas (hipo ou hiperglicemia) foram baseadas na informação do paciente. Consideraram-se excursões glicêmicas valores de glicemia abaixo de $60 \mathrm{mg} / \mathrm{dl}$ (hipoglicemia) e acima de $140 \mathrm{mg} / \mathrm{dl}$ (hiperglicemia). A hipoglicemia noturna foi definida por glicemia $<60 \mathrm{mg} / \mathrm{dl}$ no período entre $23 \mathrm{~h}$ e $6 \mathrm{~h}$.

Os níveis de glico-hemoglobina (Alc) foram determinados antes e 3 meses após o início da terapia basal com insulina glargina. Os valores de Alc foram estabelecidos pelo método HPLC, com valores de referência entre 4,3\% e 6,9\%.

Os dados foram coletados em uma ficha-padrão e analisados pelo software Minitab, através do teste $t$ de Student, médias e desvio-padrão. Os dados foram incluídos no estudo após autorização escrita dos pacientes. Não houve conflitos de interesse (patrocínio ou doações) a ser registrado neste estudo.

\section{RESULTADOS}

Foram avaliados 49 pacientes, com tempo médio de DM de $13,2 \pm 10,1$ anos, sendo $57,14 \%$ do sexo feminino e $42,86 \%$ do sexo masculino. A idade variou de 
4 a 72 anos, com média de $24,7 \pm 16,5$ anos. Houve predomínio de pacientes com DMl (93,1\%).

Quanto ao esquema prévio de insulinização, $51,2 \%$ dos pacientes utilizavam apenas insulina NPH, $10,2 \% \mathrm{NPH}$ associada à insulina regular, $6,1 \% \mathrm{NPH}$ associada à insulina aspart e $32,5 \%$ associada à insulina lispro. A insulina NPH era utilizada duas vezes ao dia nos pacientes estudados. Três meses depois, 46,9\% utilizavam apenas insulina glargina, $6,2 \%$, em uso de glargina e insulina regular, $16,3 \%$ dos pacientes usavam glargina associada à insulina aspart e 30,6\%, glargina com lispro, evidenciando um incremento do uso de insulina ultra-rápida (especialmente a aspart) em relação à insulina regular.

Quanto à dose total de insulina utilizada, verificou-se redução de $0,11 \pm 0,19 \mathrm{U} / \mathrm{kg}(\mathrm{p}<0,05)$ após três meses de tratamento. Quanto aos parâmetros clínicos (eventos glicêmicos adversos, crise hipoglicêmica grave, crises convulsivas, internações por cetoacidose), observou-se melhora significativa após o uso da insulina glargina (tabela 1). Não foi registrado nenhum novo episódio de crise convulsiva por hipoglicemia ou cetoacidose após introdução da insulina glargina $(\mathrm{p}=0,001)$.

A avaliação do controle metabólico (Alc) nos 49 pacientes após três meses do uso da insulina glargina evidenciou redução significante da Alc nesses pacientes, com redução média percentual de $1,10 \pm$ $1,46 \%$ (tabela 1 ).

Não foram observadas complicações importantes durante a utilização da terapia basal/ bólus com insulina glargina. Não houve registro de trauma local, reação alérgica, dor significativa limitante em nenhum caso. Não houve nenhum caso de inoperabilidade técnica ou abandono do tratamento.

\section{DISCUSSĀO}

O estudo realizado demonstrou impacto significativo da substituição da insulina NPH por insulina glargina em pacientes diabéticos descompensados, após três meses, com redução das crises hipoglicêmicas, crises convulsivas e cetoacidose, bem como redução dos níveis de Alc e dose diária de insulina utilizada, com boa tolerância pelos pacientes.

Os achados de redução dos eventos hipoglicêmicos graves, inexistência de novos episódios de crises convulsivas e cetoacidose, estão associados a melhor aceitação e adesão ao tratamento, o que é corroborado por Fulcher e cols., que observou redução dos eventos hipoglicêmicos severos (incluindo noturno e silencioso) em usuários de insulina glargina vs. NPH e redução significativa da Alc após 30 semanas (13).

Quanto ao impacto da insulina glargina nos níveis de Alc, ainda não há consenso na literatura. Neste estudo, após a mudança de terapêutica de $\mathrm{NPH}$ para glargina, por 12 semanas, verificou-se redução dos níveis de Alc, de acordo com os alguns autores (13-18). Além da redução da Alc, vem sendo descrita a redução dos níveis de glicemia de jejum, como demonstrado por Fulcher e cols., que obtiveram, ainda, redução de 9,2 para $8,3 \%$ da Alc (vs. NPH: 9,7 para 9,1\%) (13). Já Yokama e cols. não observaram diferença significativa nos níveis de Alc em pacientes diabéticos tipo 2 , usuários prévios de insulina NPH noturna, que receberam insulina glargina matinal 1 x dia (19).

Nesse estudo, a manutenção de níveis ainda elevados de Alc se deu, provavelmente, devido ao curto período de seguimento. Assim, a menor dose diária de insulina glargina utilizada após 3 meses pode estar associada a um controle glicêmico ainda não ideal (Alc > 7,0\%). Um maior tempo de seguimento será necessário para definir o real impacto dessas variáveis no controle metabólico de pacientes diabéticos em uso de insulina glargina. Em estudo semelhante, Yokoyama e cols. analisaram 62 pacientes com DM2 randomizados para insulina NPH noturna e glargina matinal. Verificaram um aumento da dose de insulina

Tabela 1. Parâmetros clínico-laboratoriais após três meses da substituição da insulina NPH por insulina glargina em uma coorte de pacientes diabéticos descompensados (A1c > 6,9\%).

\begin{tabular}{lccc}
\hline & Antes & Após (3 meses) & Valor $\boldsymbol{P}$ \\
Parâmetros clínicos (eventos/paciente) & & & \\
Hipoglicemia grave & $0,81 \pm 1,71$ & $0,04 \pm 0,28$ & 0,020 \\
Crise convulsiva & $0,38 \pm 1,15$ & 0 & 0,023 \\
Cetoacidose diabética & $0,08 \pm 0,34$ & 0 & 0,001 \\
Parâmetros laboratoriais & & & \\
Dose diária de insulina (U/kg) & $0,75 \pm 0,21$ & $0,64 \pm 0,19$ & 0,045 \\
A1c (mg\%) & $10,2 \pm 2,0$ & $9,1 \pm 1,8$ & 0,019 \\
\hline
\end{tabular}


basal (glargina), sem alteração significativa da dose total diária de insulina após a substituição da insulina $\mathrm{NPH}$, para se atingir os níveis glicêmicos ideais, sem alteração dos índices de hipoglicemias (19).

Já em pacientes com DM2 usuários de terapia de bomba de insulina (TBI), que passaram a utilizar insulina glargina como basal, observa-se redução semelhante da Alc, sem diferença entre os grupos (TBI vs. glargina), achados equivalentes para ganho de peso, hipoglicemias e satisfação com o tratamento (20). Em se tratando de pacientes com DMl, a dose de insulina glargina preconizada em usuários prévios de TBI tem sido considerada a dose basal usada na TBI (proporção 1:1), com manutenção de Alc dentro dos níveis ideais, sem impacto nas excursões glicêmicas (21). Esses dados reforçam a segurança para o uso da insulina glargina em usuários prévios mal controlados com NPH, como também em pacientes bem controlados em uso de TBI (mínimo de 6 meses), sem prejuízo para o controle metabólico nesses casos.

Em crianças/adolescentes com DMl, o uso da insulina glargina vem se tornando cada vez mais difundido. Na faixa etária pediátrica, a insulina glargina vem proporcionando maior estabilidade glicêmica, com redução da glicemia de jejum, dos níveis de Alc, redução da dose diária de insulina total $(0,9$ para 0,83 $\mathrm{U} / \mathrm{kg} / \mathrm{dia}, \mathrm{p}<0,05)$ e das crises de hipoglicemia severa, em consonância com os dados aqui apresentados. Em menores de 6 anos, há elevada incidência de hipoglicemia noturna grave, com sérios danos cerebrais descritos. A principal vantagem dessa insulina basal (glargina) tem sido a redução das crises de hipoglicemia noturna, incluindo hipoglicemia silenciosa (assintomática). Não parece haver diferença quanto aos níveis de glicemia de jejum e Alc em relação à terapia prévia com NPH, como comprovado em estudo com 128 crianças por Dixon e cols. (22).

Em pacientes com DM2 em uso prévio de antidiabéticos orais (ADO), a introdução da insulina glargina $1 \mathrm{x}$ dia também parece promover resultados satisfatórios, de acordo com os dados da literatura. O acompanhamento de 198 diabéticos tipo 2 que receberam associação de $\mathrm{ADO}$ (usados previamente) com insulina glargina $1 \mathrm{x}$ dia, mostrou redução significativa dos níveis de Alc $(9,4$ a $8,4 \%)$, sem aumento dos eventos hipoglicêmicos ou ganho de peso significativo (16).

Quanto ao impacto da introdução da insulina glargina em pacientes diabéticos usuários de NPH e mal controlados, verificou-se melhora do controle metabólico, redução dos eventos glicêmicos adversos, com boa tolerância pelos pacientes. Tal fato é confirmado pela literatura através de uma satisfação de cerca de $90 \%$ dos pacientes em uso de insulina glargina, relacionada à maior estabilidade clínica, menor número de internações, maior flexibilidade/ conforto, com maior adesão ao tratamento $(14,18)$.

Quanto aos custos, estudo americano (Califórnia) mostra uma redução das crises hipoglicêmicas e eventos hiperglicêmicos graves, com redução das internações e visitas aos pronto-atendimentos, culminando com redução média de 185 para 72 dólares por paciente em relação ao custo para o sistema público (23). Novos estudos, principalmente no Brasil, são necessários para determinar tal redução de custos, inclusive como justificativa para padronização da insulina glargina no sistema público de saúde.

Algumas limitações do estudo devem ser enfatizadas, uma vez que se trata de estudo observacional, não-randomizado, não-controlado, de casos tratados em unidade de referência. Consideramos, pois, que os achados não podem ser generalizados e algumas particularidades, como o entusiasmo dos pacientes com novo tratamento, empenho da equipe em educar e obter bons resultados, são capazes de modificar os resultados e não são passíveis de controle.

Outra limitação desse estudo reside no fato de um grande número de pacientes ter recebido apenas insulina glargina uma vez ao dia, contribuindo possivelmente para a redução da dose diária de insulina e níveis ainda elevados de Alc ao final dos três meses. Segundo os estudos "Treat to Target" e "LANMET", a melhora do controle glicêmico esteve relacionada a um incremento da dose diária total de insulina, com redução significativa dos níveis de Alc $(12,24)$. O acesso ao sistema público de saúde e altos custos no tratamento parecem ter sido a principal dificuldade no uso de doses de insulina ultra-rápida nas refeições nesses pacientes. Contudo, este trabalho representa a prática clínica real com essa nova terapia, a experiência do dia-a-dia de um grupo e não de estudos clínicos randomizados.

\section{CONCLUSŌES}

A substituição da insulina NPH por insulina glargina em uma coorte de pacientes diabéticos descompensados foi segura, bem tolerada na prática clínica diária, com redução da Alc, de crises hipoglicêmicas, de crises convulsivas e da cetoacidose, após 3 meses. Estima-se que a melhora clínica evidenciada facilite a adesão ao tratamento e melhora da qualidade de vida dos pacientes. $\mathrm{O}$ uso da insulina glargina em pacientes com DM2, crianças com DMl, usuários prévios de 
TBI, tem se mostrado eficaz na literatura. Novos estudos em relação aos custos são necessários para padronização dessa insulina no sistema público de saúde.

\section{REFERÊNCIAS}

1. Brasil. Ministério da Saúde. Departamento de Assistência e Promoção à Saúde. Secretaria de Assistência à Saúde. Manual de Diabetes. 2a ed. revista. Brasília: Codeg, 1994.

2. Koro CE, Bowlin SJ, Bourgeois N, Fedder DO. Glycemic control from 1988 to 2000 among US adults diagnosed with type 2 diabetes: a preliminary report. Diabetes Care 2004;27:1720.

3. American Diabetes Association. Standards of medical care for patients with diabetes mellitus. Diabetes Care 2003;26(suppl 1):S33-50.

4. Malerbi D, Damiani D, Rassi N. Posição de consenso da Sociedade Brasileira de Diabetes: insulinoterapia intensiva e terapêutica com bombas de insulina. Arq Bras Endocrinol Metab 2006;50(1):125-35.

5. Diabetes Control and Complications Trial Research Group. The effect of intensive treatment of diabetes on the developmen and progression of long-term complications in insulin-dependent diabetes mellitus. N Engl J Med 1993,329:977-86.

6. Peters A, Klose O, Hefty R, Keck F, Kerner W. The influence of insulin antibodies on the pharmacokinetics of NPH insulin in patients with type 1 diabetes treated with human insulin. Diabet Med 1995; 12:925-30.

7. Siepke G, Geisen K, Neubauer HP, Pittius C, Pittius C, Rosskamp R, et al. New insulin preparations with prolonged action profiles: A21-modified arginine insulins. Diabetologia 1992;suppl 1:A4.

8. Rosskamp R, Park G. Long-acting insulin analogs. Diabetes Care 1999;22(suppl. 2):B109-13.

9. Coates PA, Mukherjec S, Luzio S, Srodzinski KA, Kurzhals $R$, Rosskamp R, et al. Pharmacokinetics of a long-acting human insulin analogue (HOE901) in healthy subjects. Diabetes 1995;44(suppl 1):130A.

10. Soon PC, Matthews DR, Rosskamp R, Herz M, Kurzhals R Profile of action of biosynthetic long acting insulin (HOE901) tested in normal volunteers by glucose clamp methodology. Diabetes 1997:46(1):161A.

11. Dreyer M, Pein M, Schimidt C, Heidtmann B, Schlunzen $M$, Rosskamp D. Comparison of the pharmacokinetics/dynamics of Gly(A21)-Arg(B31,B32)-human insulin (HOE71GT) with $\mathrm{NPH}$-human insulin following subcutaneous injection by using euglycaemic clamp technique. Diabetologia 1998;37(suppl 1):A62.

12. Yki-Jarvinen $H$, Kauppnen-Makelin $R$, Tiikainen $M$, Vahatolo $\mathrm{M}$, Virtamo $\mathrm{H}$, Nikkila K, et al. Insulin glargine or NPH combined with metformin in type 2 diabetes: The LANMET study. Diabetologia 2006;49:442-51.

13. Fulcher GR, Gilbert RE, Yue DK. Glargine is superior to neutral protamine Hagedorn for improving glycated haemoglobin and fasting blood glucose levels during intensive insulin therapy. Intern Med J 2005;35:536-42.
14. Heljic B, Velija-Asimi Z, Burekovic A, Buturovic B, Ceric A, Horozic B, et al. LEAP (Lantus Early Access Programme) in Sarajevo. Med Arh 2005;59(5):283-5.

15. Colino E, Lopez-Capape M, Golmayo L, Alvarez MA, Alonso $M$, Barrio R. Therapy with insulin glargine (Lantus) in toddlers, children and adolescents with type 1 diabetes. Diabetes Res Clin Pract 2005;70:1-7.

16. Kacerovsky-Bielesz G, Dressler A, Freunscht R. Long-term glycaemic control with insulin glargine in type 2 diabetes. Diabetes Res Clin Pract 2005;17:1-7.

17. Donaubauer B, Schneider K, Schweitzer MA. Introduction of insulin glargine to basal-bolus therapy improves metabolic control in patients with type 1 diabetes in everyday clinical practice. Diabetologia 2005;48(suppl 1):abs 905.

18. Rosak C, Jung R, Nuber U, Schweitzer MA, Hofman U. The impact of insulin glargine on lifestyle flexibility in patients with type 1 diabetes. Diabetologia 2005;48(suppl 1):abs 904.

19. Yokoyama H, Tada J, Kamikawa F, Kanno S, Yokota $Y$, Kuramitsu M. Efficacy of conversion from bedtime NPH insulin to morning insulin Glargine in type 2 diabetic patients on basal-prandial insulin therapy. Diabetes Res Clin Pract 2006; $73: 35-40$.

20. Herman $\mathrm{WH}$, llag $\mathrm{LL}$, Johnson $\mathrm{SL}$, Martin $\mathrm{CL}$, Sinding J, Al Harthi $A$, et al. A clinical trial of continuous subcutaneous insulin infusion versus multiple daily injections in older adults with type 2 diabetes. Diabetes Care 2005;28:1568-73.

21. Bode BW, Steed RD, Schleusener DS, Strange P. Switch to multiple daily injections with insulin Glargine and insulin lispro from continuous subcutaneous insulin infusion with insulin lispro: a randomized, open-label study using a continuous glucose monitoring system. Endocr Pract 2005; 11:157-64.

22. Dixon B, Peter Chase $H$, Burdick J, Fiallo-Scharer R, Walravens $P$, Klingensmith $G$, et al. Use of insulin glargine in children under age 6 with type 1 diabetes. Pediatr Diabetes 2005;6(3):150-4.

23. Zhang $Q$, Menditto L. Incremental cost savings 6 months following initiation of insulin glargine in a Medicaid fee-for-service sample. Am J Ther 2005;12(4):337-43.

24. Riddle MC, Rosenstock J, Gerich J; Insulin Glargine 4002 Study Investigators. The treat-to-target trial: randomized addition of glargine or human NPH insulin to oral therapy of type 2 diabetic patients. Diabetes Care 2003;26:3080-6.

Endereço para correspondência:

Frederico Fernandes Ribeiro Maia

Rua Nunes Vieira 299, ap. 702

30350-120 Belo Horizonte, MG

E-mail: fredfrm@hotmail.com / frederico@diabetes.med.br 